

- Additional material is published online only. To view please visit the journal online (http://dx.doi.org/10.1136/ijgc2020-001299)

Department of Obstetrics and Gynecology, Institute of Women's Life Medical Science, Yonsei University College of Medicine, Seoul, Korea

Correspondence to Professor Jung-Yun Lee, Department of Gynecology and Obstetrics, Institute of Women's Life Medical Science, Yonsei University College of Medicine, Seoul, Korea; yodrum682@ gmail.com

Received 14 February 2020 Revised 28 May 2020 Accepted 2 June 2020 Published Online First 15 July 2020

\title{
Impact of subcutaneous negative pressure drains on surgical wound healing in ovarian cancer
}

Young Shin Chung, Jung-Yun Lee (D), Eun Ji Nam, Sunghoon Kim, Sang Wun Kim, Young Tae Kim

\section{HIGHLIGHTS}

- Subcutaneous negative pressure drains were associated with fewer overall wound complications.

- Subcutaneous negative pressure drains were associated with reduced seroma formation.

- Subcutaneous drains were identified as a predictive factor for preventing wound complications.

\begin{abstract}
Introduction Subcutaneous negative pressure wound drains have been used to reduce wound complication rates in various surgical procedures. However, research on the benefits of subcutaneous drains on wound healing after ovarian cancer surgery is limited. The aim of this study was to assess the effects of subcutaneous negative pressure drains on wound healing after abdominal surgery for ovarian cancer.
\end{abstract}

Methods Patients who underwent surgery with a midline incision for ovarian cancer between February 2015 and May 2019 were retrospectively examined. Patients were divided into two groups according to the presence (group $1 ; n=99$ ) or absence (group 2; $n=213$ ) of subcutaneous wound drains. The primary endpoint was the incidence of wound complications within 8 weeks after abdominal surgery. The secondary endpoints were time interval from surgery to adjuvant chemotherapy and survival.

Results Patients in group 1 were older (mean 58.5 vs 55.4 years; $p=0.02$ ), and had higher rates of previous abdominal surgery ( $66.7 \%$ vs $47.9 \%$; $p=0.002)$, bowel surgery ( $47.5 \%$ vs $34.3 \%$; $p=0.026)$, and had a high surgical complexity score ( $53.5 \%$ vs $33.8 \% ; p<0.001)$ compared with patients in group 2 . Median body mass index was not different between the two groups: group $1,22.9 \mathrm{~kg} / \mathrm{m}^{2}$ (range 16.0 to 33.3 ) and group 2, $22.8 \mathrm{~kg} /$ $\mathrm{m}^{2}$ (range 16.4 to 37.5$)(\mathrm{p}=0.858)$. A higher rate of clear wound healing $(82.8 \%$ vs $71.8 \% ; p=0.036)$ and a lower rate of seroma formation $(6.1 \%$ vs $16.0 \% ; p=0.015)$ were observed in group 1 compared with group 2 . After multivariate analysis, subcutaneous wound drain placement was identified as an independent predictive factor for preventing wound complications (adjusted odds ratio $0.43 ; 95 \%$ confidence interval 0.21 to 0.87 ). Time interval from surgery to adjuvant treatment was significantly longer in patients with wound complications than in those with clear wound healing (mean 23.6 vs 19.2 days; $p=0.003$ ). Kaplan-Meier analysis, however, showed no significant differences in progression free or overall survival between the two groups $(p=0.35$ and $p=0.96$, respectively).

Conclusion The prophylactic use of subcutaneous negative pressure drains after abdominal surgery for ovarian cancer significantly reduced the incidence of wound complications in this study.

\section{INTRODUCTION}

Patients undergoing surgery for gynecologic cancer experience wound complications at rates ranging from $5 \%$ to $35 \%{ }^{12}$ Wound complications lead to extended stays in hospital, worse quality of life, and increased treatment costs. ${ }^{34}$ In ovarian cancer specifically, wound complications have been shown to be associated with increased readmission rates and postoperative mortality, and delays in chemotherapeutic treatment after abdominal surgery. ${ }^{5-7}$

Subcutaneous wound drains were developed approximately two decades ago to drain transudate from wounds. ${ }^{8}$ These drains reduce potential deadspace in the subcutaneous tissue by preventing the accumulation of transudate from surgical wounds. ${ }^{89}$ Subcutaneous wound drains have shown promising results in various types of surgery. ${ }^{8-19}$ However, the value of wound drains in gynecological surgery, ${ }^{8131617}$ as in cesarean delivery, ${ }^{914}{ }^{15}$ is still controversial. Most studies of wound drains in surgeries for gynecological malignancies are limited by the inclusion of only a small number of patients, including those with gynecological benign diseases. ${ }^{8}{ }^{13}$ Moreover, except for one study, there is no information on the efficacy of wound drains in gynecological malignancy only ${ }^{17}$ : in the aforementioned study, only a small number of patients were included in the no drain group, and clinical benefits were not exhaustively evaluated.

We aimed to evaluate the effectiveness of subcutaneous negative pressure drains for wound healing in a large cohort of patients who underwent abdominal surgery for ovarian cancer. We also explored several clinical benefits, such as time interval from surgery to adjuvant treatment and survival outcomes. 


\section{Original research}

\section{METHODS}

\section{Patient Population}

Medical records of patients who underwent abdominal surgery with a midline incision for ovarian cancer at a single institution between February 2015 and May 2019 were retrospectively reviewed. All patients underwent primary debulking surgery or interval debulking surgery after neoadjuvant chemotherapy. The following patients were excluded from the study: patients diagnosed with nonepithelial ovarian cancer; those who received transverse incisions during surgery; and those who underwent secondary debulking surgery owing to disease recurrence (online supplementary Figure S1). All inpatient and outpatient follow-up medical records were reviewed. The study was conducted with the approval of the institutional review board of Severance Hospital, Yonsei University College of Medicine (No 4-2019-0900). The requirement for written informed consent was waived owing to the retrospective nature of this study.

\section{Surgical Protocol}

Based on the presence or absence of subcutaneous wound drains, patients were divided into two groups: group 1 comprised patients who received wound drains and group 2 comprised those without wound drains. All patients received the same preoperative, operative, and postoperative management, except for wound drain placement, during surgery. Except for wound drain placement, all surgical procedures were performed by one of five gynecologic oncology surgeons according to the standard protocol at our institution. Because placement of wound drains was at the surgeon's discretion, all were placed by a single surgeon. Therefore, all surgical procedures in group 1 were performed by a single surgeon (J-YL). However, he was not involved in the surgical procedures in group 2.

Preoperative prophylactic antibiotics with $1 \mathrm{~g}$ of a second generation cephalosporin were intravenously administered within 60 min of the incision. After surgery, antibiotic prophylaxis with $1 \mathrm{~g}$ of a second generation cephalosporin, $500 \mathrm{mg}$ of metronidazole, and $400 \mathrm{mg}$ of aminoglycoside were allowed intravenously for $5-7$ days. All patients received preoperative bowel preparation, which consisted of $4 \mathrm{~L}$ of polyethylene glycol solution. For venous thromboembolic prophylaxis, sequential compression devices with low molecular weight heparin injections were used. Pubic hair was not removed before surgery, and in all patients an abdominal scrub at the surgical site was performed using povidone-iodine.

Low midline incisions were made from the symphysis pubis, around the umbilicus, and extending to the xiphoid process if upper abdominal surgical procedures were needed. Surgical incisions were made using a scalpel and cutting electrocautery. The abdominal fascia was sutured interruptedly (approximately $1 \mathrm{~cm}$ apart) after all surgical procedures were completed. During skin closure using staples, a subcutaneous wound drain was secured to the skin 2-3 cm away from the surgical incision, and subcutaneous tissue closure was not performed.

In group 1, Jackson-Pratt drains composed of perforated silicone round tubes with an inner diameter of $1.6 \mathrm{~mm}$ and an outer diameter of $3.2 \mathrm{~mm}$ were placed in the subcutaneous space, exiting through a separate stab surgical incision. The drains were connected to a $200 \mathrm{~mL}$ silicone bulb reservoir that continuously removed subcutaneous discharge using negative pressure (BAROVAC SS200M; Sewoon Medical, Cheonan-si, Chungcheongnam-do, Republic of Korea). As subcutaneous tissue closure was not performed, the skin was closed using stainless steel staples. An illustration of subcutaneous wound drain insertion is presented in Figure 1. The drains were left in place until $<1 \mathrm{~mL}$ of drainage was observed daily. In contrast, group 2 did not receive subcutaneous wound drains. The subcutaneous tissue was closed with an interrupted suture, and the skin was approximated with staples.

Adhesive closure strips and gauze were applied to the skin surface of all surgical wounds and were removed on the morning of the third postoperative day. Afterward, the wound was left uncovered and a povidone-iodine dressing was applied daily. Staples remained in the skin for at least 7 days after surgery. Before wound dressing, all wounds were assessed by inspection and palpation of the incision and surrounding area carefully to identify any wound complications. Ultrasound or computed tomography was not used to measure or evaluate wound complications.


Figure 1 Subcutaneous negative pressure wound drain placement. (A) A round Jackson-Pratt drain was inserted within the subcutaneous tissue, exiting through a separate stab surgical incision. (B) Subcutaneous tissue closure was not performed, and the skin was closed using stainless steel staples. 
Patients received three cycles of neoadjuvant chemotherapy consisting of a taxane and platinum based regimen before interval debulking surgery. Surgeries were performed to achieve complete cytoreduction leaving no gross residual disease. We defined bowel surgeries as small bowel resection, low anterior resection, and total/ partial colectomy. Simple appendectomy, bowel adhesiolysis, and tumor resection of the bowel were not considered bowel surgeries. The complexity of the surgery was classified into three groups according to the surgical complexity score: low $(\leq 3)$, intermediate (4-7), and high $(\geq 8) .{ }^{20}$ Thirty-four patients underwent interval debulking surgery following hyperthermic intraperitoneal chemotherapy, as indicated in the standard protocol of our institution. ${ }^{21}$

\section{Outcome Evaluation}

Wound complications within 8 weeks after ovarian cancer surgeries were assessed during wound dressing, staple removal, and outpatient clinic visits following discharge. All wound complications of both inpatients and outpatients were recorded.

Clear wound healing was defined as an absence of complications during surgical wound healing. Wound complications were defined as follows: (1) infection, positive wound culture, and/or redness, tenderness, induration, fever, and purulent discharge at the surgical site; (2) dehiscence, an open wound of $>1 \mathrm{~cm}$, regardless of the reason; (3) seroma, a collection of serous fluid underneath the surgical incision; (4) hematoma, formation of blood clots; and (5) reoperation, repeat surgery owing to wound complications.

\section{Endpoints and Statistical Analysis}

The primary endpoint of the study was the incidence of wound complications within 8 weeks after abdominal surgery. The secondary endpoints were time interval from surgery to adjuvant chemotherapy and survival rate. Descriptive statistics were calculated for all variables to determine the differences between the two groups. The Student's t test or the Mann-Whitney U test were used for continuous variables, and Pearson's $\chi^{2}$ test or Fisher's exact test were used for categorical variables. Multivariate logistic regression analysis was performed to identify independent factors correlated with wound complications. All factors found to be significant in the univariate analysis were included in multivariate analysis. Progression free survival or overall survival was compared using Kaplan-Meier curves between the groups and were tested using the log rank test. We performed statistical analyses on a personal computer with Statistical Package for the Social Sciences software (V.25.0, IBM Corp, Armonk, NY, USA). A two tailed p value $<0.05$ was regarded as statistically significance.

\section{RESULTS}

\section{Patient Characteristics}

Among the 312 patients included, 99 and 213 patients were allocated to group 1 and group 2, respectively (online supplementary Figure S1). The baseline characteristics of the patients from both groups are presented in Table 1. There was no difference in median body mass index between the two groups: group $1,22.9 \mathrm{~kg} / \mathrm{m}^{2}$ (range 16.0 to 33.3 ) and group $2,22.8 \mathrm{~kg} / \mathrm{m}^{2}$ (16.4 to 37.5$)(\mathrm{p}=0.86)$. However, there were significant differences between groups 1 and 2 with respect to age (mean 58.5 vs 55.4 years; $p=0.020$ ), poor performance status (American Society of Anesthesiologists score

\begin{tabular}{|c|c|c|c|}
\hline Characteristic & $\begin{array}{l}\text { With wound } \\
\text { drain } \\
\text { (group 1, } \\
\mathrm{n}=99 \text { ) }\end{array}$ & $\begin{array}{l}\text { Without } \\
\text { wound drain } \\
\text { (group 2, } \\
\mathrm{n}=213 \text { ) }\end{array}$ & $\begin{array}{l}P \\
\text { value }\end{array}$ \\
\hline $\begin{array}{l}\text { Age at surgery } \\
\text { (years) (mean } \pm S D \text { ) }\end{array}$ & $58.5 \pm 9.7$ & $55.4 \pm 11.5$ & \\
\hline $\begin{array}{l}\text { BMl at surgery }(\mathrm{kg} / \\
\left.\mathrm{m}^{2}\right)(\mathrm{mean} \pm \mathrm{SD})\end{array}$ & $23.2 \pm 3.5$ & $23.3 \pm 3.6$ & \\
\hline \multicolumn{4}{|l|}{$\begin{array}{l}\text { Comorbidities (n } \\
(\%))\end{array}$} \\
\hline Hypertension & $23(23.2)$ & $52(24.4)$ & 0.820 \\
\hline Diabetes mellitus & $10(10.1)$ & $14(6.6)$ & 0.276 \\
\hline Smoking & $2(2.0)$ & $1(0.5)$ & 0.191 \\
\hline ASA score (n (\%)) & & & 0.001 \\
\hline 1 & $10(10.1)$ & $51(23.9)$ & \\
\hline 2 & $45(45.5)$ & $106(49.8)$ & \\
\hline 3 & $44(44.4)$ & 55 (25.8) & \\
\hline 4 & $0(0)$ & $1(0.5)$ & \\
\hline $\begin{array}{l}\text { Previous abdominal } \\
\text { surgery }(\mathrm{n}(\%))\end{array}$ & $66(66.7)$ & $102(47.9)$ & 0.002 \\
\hline $\begin{array}{l}\text { Previous radiation } \\
(\mathrm{n}(\%))\end{array}$ & $2(2.0)$ & $2(0.9)$ & 0.594 \\
\hline
\end{tabular}

BMI, body mass index; ASA, American Society of Anesthesiologists.

$\geq 3,44.4 \%$ vs $26.3 \% ; p=0.001$ ), and previous abdominal surgery (66.7\% vs $47.9 \% ; p=0.002)$.

Surgical and pathologic characteristics are summarized in Table 2. There were significant differences between groups 1 and 2 with respect to the percentage of patients who underwent neoadjuvant chemotherapy ( $48.5 \%$ vs $25.4 \%$; $p<0.001)$, bowel surgery (47.5\% vs $34.3 \% ; p=0.026)$, high surgical complexity score surgery (score $\geq 8,53.5 \%$ vs $33.8 \% ; p<0.001$ ), and hyperthermic intraperitoneal chemotherapy $(31.3 \%$ vs $1.4 \% ; p<0.001)$. The duration of surgery (mean 481.7 vs $425.9 \mathrm{~min} ; \mathrm{p}=0.008$ ) and estimated blood loss (mean 1574.9 vs $1190.2 \mathrm{~mL} ; \mathrm{p}=0.043$ ) were also statistically different between groups 1 and 2 .

The mean duration of postoperative prophylactic antibiotics was $6.6 \pm 1.6$ days in the whole cohort, with differences between groups 1 and 2 (mean 6.1 vs 6.8 days; $p=0.031$ ). The median follow-up period was 21.8 months (range 2.1 to 54 ).

\section{Wound and Clinical Outcomes}

Low and extended midline incisions were performed in 10 and 302 patients, respectively. There were no associations between wound complications and length of surgical incision $(30.0 \%$ vs $24.6 \%$; $\mathrm{p}=0.71$ ). The mean duration of wound drainage and amount of fluid drained were $8.8 \pm 2.9$ days and $117.3 \pm 101.4 \mathrm{~mL}$, respectively.

The wound complications that occurred in both groups are described in Table 3. The clear wound healing rates in groups 1 and 2 were $82.8 \%(n=82)$ and $71.8 \%(n=213)$, respectively $(p=0.036)$. The incidence of overall wound complications was $17.2 \%$, the majority of which were wound dehiscence and seroma. The rate of seroma formation was significantly lower in group 1 than in group 


\section{Original research}

Table 2 Surgical and pathologic characteristics of the patients

\begin{tabular}{|c|c|c|c|}
\hline Characteristic & $\begin{array}{l}\text { With wound drain } \\
\text { (group 1, } \mathrm{n}=99 \text { ) }\end{array}$ & $\begin{array}{l}\text { Without wound drain } \\
\text { (group 2, } n=213 \text { ) }\end{array}$ & $P$ value \\
\hline FIGO stage (n (\%)) & & & 0.072 \\
\hline I & $6(6.1)$ & $18(8.5)$ & \\
\hline II & $6(6.1)$ & $9(4.2)$ & \\
\hline III & $32(32.3)$ & $98(46.0)$ & \\
\hline IV & $55(55.6)$ & $88(41.3)$ & \\
\hline \multicolumn{4}{|l|}{ Histology (n (\%)) } \\
\hline HGSC & $71(71.7)$ & $149(72.3)$ & 0.983 \\
\hline Non-HGSC & $28(28.3)$ & $57(27.7)$ & \\
\hline NAC & $48(48.5)$ & $54(25.4)$ & $<0.001$ \\
\hline Bowel surgery (n (\%)) & 47 (47.5) & 73 (34.3) & 0.026 \\
\hline Surgical complexity group* (n (\%)) & & & $<0.001$ \\
\hline Low & $4(4.0)$ & $1(0.5)$ & \\
\hline Intermediate & $42(42.4)$ & $140(65.7)$ & \\
\hline High & $53(53.5)$ & $72(33.8)$ & \\
\hline Residual disease (n (\%)) & & & 0.833 \\
\hline No gross residual & $54(55.1)$ & $106(53.8)$ & \\
\hline$>0 \mathrm{~cm}$ & $44(44.9)$ & $91(46.2)$ & \\
\hline Not available & 1 & 16 & \\
\hline Adhesiolysis (n (\%)) & $71(71.7)$ & $129(60.6)$ & 0.056 \\
\hline HIPEC (n (\%)) & $31(31.3)$ & $3(1.4)$ & $<0.001$ \\
\hline Duration of surgery (min) (mean $\pm S D)$ & $481.7 \pm 186.9$ & $425.9 \pm 165.3$ & \\
\hline Estimated blood loss (mL) (mean \pm SD) & $1574.9 \pm 2095.7$ & $1190.2 \pm 1235.3$ & \\
\hline
\end{tabular}

${ }^{*}$ According to Aletti et al. ${ }^{20}$

FIGO, International Federation of Gynecology and Obstetrics; HGSC, high grade serous carcinoma; HIPEC, hyperthermic intraperitoneal chemotherapy; NAC, neoadjuvant chemotherapy.

$2(6.1 \%$ vs $16.0 \% ; p=0.015)$. There were no statistically significant differences in infection $(3.0 \%$ vs $3.8 \%$; $p=1.0)$, dehiscence $(11.1 \%$ vs $16.0 \% ; p=0.26)$, hematoma $(2.0 \%$ vs $0 \% ; p=0.10)$, or reoperation $(4.0 \%$ vs $2.3 \% ; p=0.47)$ between groups 1 and 2 . KaplanMeier analysis showed no significant differences in progression free survival or overall survival between the two groups $(p=0.88$ and $p=0.32$, respectively) (online supplementary Figure $S 2$ ).

Table 3 Wound outcomes according to the presence of a wound drain

\begin{tabular}{|c|c|c|c|}
\hline Outcome & $\begin{array}{l}\text { With wound } \\
\text { drain } \\
\text { (group 1, } \\
\mathrm{n}=99 \text { ) }\end{array}$ & $\begin{array}{l}\text { Without } \\
\text { wound } \\
\text { drain } \\
\text { (group 2, } \\
\mathrm{n}=213 \text { ) }\end{array}$ & $P$ value \\
\hline Clear healing (n (\%)) & $82(82.8)$ & 153 (71.8) & 0.036 \\
\hline Infection (n (\%)) & $3(3.0)$ & $8(3.8)$ & 1.000 \\
\hline Dehiscence (n (\%)) & $11(11.1)$ & $34(16.0)$ & 0.256 \\
\hline Seroma (n (\%)) & $6(6.1)$ & $34(16.0)$ & 0.015 \\
\hline Hematoma (n (\%)) & $2(2.0)$ & $0(0)$ & 0.100 \\
\hline Reoperation (n (\%)) & $4(4.0)$ & $5(2.3)$ & 0.472 \\
\hline
\end{tabular}

In contrast, the duration of hospital stay (mean 20.5 vs 17.1 days; $p=0.008$ ) and the time interval from surgery to adjuvant chemotherapy (mean 23.6 vs 19.2 days; $p=0.003$ ) were significantly longer among patients with wound complications than among patients with clear wound healing (Online supplementary table S1). Moreover, Kaplan-Meier analysis showed no significant differences in progression free survival or overall survival between patients with wound complications and those with clear wound healing ( $p=0.35$ and $p=0.96$, respectively) (online supplementary Figure S3).

\section{Predictors of Wound Complications}

The clinical factors associated with wound complications are listed in Table 4. After multivariate logistic regression analysis, placement of subcutaneous wound drains was identified as an independent predictive factor for reduced wound complications (adjusted odds ratio (OR) $0.43 ; 95 \%$ confidence interval $(\mathrm{Cl}) 0.21$ to $0.87 ; p=0.02$ ), especially seroma formation (adjusted $\mathrm{OR} 0.29 ; 95 \% \mathrm{Cl} 0.10$ to $0.82 ; p=0.02$ ). Other risk factors significantly associated with wound complications were body mass index $\geq 25 \mathrm{~kg} / \mathrm{m}^{2}$ (adjusted OR 2.80; 95\% $\mathrm{Cl} 1.50$ to 5.23; $\mathrm{p}=0.001$ ), adhesiolysis (adjusted OR 2.53; $95 \% \mathrm{Cl}, 1.35$ to $4.74 ; \mathrm{p}=0.004$ ), and bowel surgery (adjusted OR 2.14; $95 \%$ Cl 1.13 to $4.07 ; p=0.020$ ). 
Table 4 Clinical factors associated with wound complications in multivariate logistic regression analysis

\begin{tabular}{|c|c|c|c|c|c|c|}
\hline \multirow[b]{2}{*}{ Variables } & \multicolumn{2}{|l|}{$\begin{array}{l}\text { Overall } \\
\text { complication }\end{array}$} & \multicolumn{2}{|l|}{ Dehiscence } & Seroma & \multirow[b]{2}{*}{$P$ value } \\
\hline & aOR (95\% Cl) & $P$ value & aOR (95\% Cl) & $P$ value & aOR (95\% Cl) & \\
\hline $\mathrm{BMI} \geq 25 \mathrm{~kg} / \mathrm{m}^{2}$ & 2.80 (1.50 to 5.23$)$ & 0.001 & $1.83(0.89$ to 3.76$)$ & 0.100 & 1.80 (0.83 to 3.89$)$ & 0.137 \\
\hline Diabetes mellitus & 1.04 (0.39 to 2.76$)$ & 0.946 & 0.47 (0.10 to 2.17$)$ & 0.331 & 1.86 (0.62 to 5.61$)$ & 0.273 \\
\hline Adhesiolysis & 2.53 (1.35 to 4.74$)$ & 0.004 & 1.79 (0.84 to 3.82$)$ & 0.131 & 2.00 (0.91 to 4.37$)$ & 0.084 \\
\hline Bowel surgery & 2.14 (1.13 to 4.07$)$ & 0.020 & 1.12 (0.52 to 2.40$)$ & 1.122 & 1.31 (0.59 to 2.93 ) & 0.512 \\
\hline HIPEC & 1.76 (0.56 to 5.57$)$ & 0.336 & 0.40 (0.08 to 1.95$)$ & 0.257 & 1.79 (0.39 to 8.20$)$ & 0.452 \\
\hline $\begin{array}{l}\text { High surgical } \\
\text { complexity }\end{array}$ & 1.49 (0.62 to 3.54$)$ & 0.347 & 1.18 (0.54 to 2.57$)$ & 0.680 & $1.28(0.44$ to 3.75$)$ & 0.648 \\
\hline $\begin{array}{l}\text { Duration of surgery } \geq 7 \\
\text { hours }\end{array}$ & 1.61 (0.62 to 4.18$)$ & 0.331 & 1.27 (0.54 to 2.95$)$ & 0.586 & 1.48 (0.46 to 4.70$)$ & 0.511 \\
\hline Wound drain & $0.43(0.21$ to 0.87$)$ & 0.020 & 0.71 (0.32 to 1.59$)$ & 0.406 & 0.29 (0.10 to 0.82$)$ & 0.020 \\
\hline
\end{tabular}

aOR, adjusted odds ratio; BMI, body mass index; HIPEC, hyperthermic intraperitoneal chemotherapy.

\section{DISCUSSION}

We found that subcutaneous negative pressure drains were associated with a reduced risk of wound complications, especially seroma formation, after abdominal surgery for ovarian cancer. Subcutaneous negative pressure wound drains have been well studied and broadly implemented for wound healing, although their placement is not included in the current guidelines. Recently, subcutaneous negative pressure wound drains have been routinely used to facilitate various surgical procedures, including thyroid surgery, axillary lymph node resection in breast cancer, breast reduction or augmentation, femoral wounds, and hip and knee joint replacement. ${ }^{10-12}$

Negative pressure wound therapy is another technique similar in principle to subcutaneous negative pressure wound drains. Since its implementation in the late 1990s, negative pressure wound therapy has been used to manage wounds effectively. ${ }^{22}{ }^{23}$ The device consists of a closed, sealed system constructed of open pore polyurethane foam, an adhesive cover, and a vacuum pump that generates negative pressure and aspirates fluid from the wound. However, these devices are expensive, and subcutaneous wound drains have an advantage in terms of cost effectiveness compared with negative pressure wound therapy.

Nevertheless, the benefits of subcutaneous negative pressure drains remain controversial in gynecological surgeries. ${ }^{8} 131617$ This may be because of differences across studies with respect to cases, patient selection, and risk factor definitions. In addition, there is currently a lack of information regarding the benefits of wound drains in patients with gynecological oncology, especially those with ovarian cancer. In 2015, Kim et $\mathrm{al}^{17}$ reported that placement of subcutaneous wound drains, the same drains used in our study, was associated with lower risks of wound disruption (OR $0.367 ; 95 \% \mathrm{Cl}, 0.145$ to $0.929 ; p=0.034$ ) and wound infection (OR $0.198 ; 95 \% \mathrm{Cl} 0.068$ to $0.582 ; \mathrm{p}=0.003$ ) after surgery in ovarian cancer. However, only a small number of patients were included in the no drain group ( $n=37)$. In our study, we found that subcutaneous drains reduced wound complications after ovarian cancer surgery, which are consistent with the results of the study of Kim et al. ${ }^{17}$ Moreover, we noted similar incidences of overall wound complications (17.2\% vs $19.0 \%$ ) and dehiscence $(11.1 \%$ vs $19.0 \%$ in the wound drain groups to those of Kim et al. However, our study had lower frequencies of infection (3.0\% vs $12.9 \%)$ and reoperation (4.0\% vs $14.7 \%)$ compared with their study, ${ }^{17}$ which may be due to improvement in disinfection or wound management over the years or the lower frequency of bowel surgeries in our population.

In this study, subcutaneous negative pressure wound drains were significantly associated with fewer overall wound complications, especially seroma formation. Seroma is a common postoperative complication, which can separate the surgical incision and leave it vulnerable to wound infection, because bacteria could access deeper layers and multiply without being suppressed in the stagnant fluid. This results in prolonged wound healing, persistent pain, prolonged hospitalization, and multiple outpatient visits. Therefore, prevention of seroma formation can reduce many other wound complications. Seroma can be detected by inspection and palpation of the wound. An asymptomatic seroma is naturally resolved in a short time without invasive treatment. Therefore, it is recommended not to aspirate asymptomatic seroma, which may increase the risk of infection. However, clinically significant and complicating infected seroma, which appears to be a symptom of the patient, should be treated.

Our results showed significantly more blood loss during surgery and prolonged hospital stay than other studies. ${ }^{24-26}$ At our institution, we performed high surgical complexity score surgeries to decrease residual disease during the study period, which may have affected the estimated blood loss. In addition, due to the high quality of health insurance, the cost of hospitalization is low in Korea. Therefore, most patients received adjuvant chemotherapy after surgical wound healing during their hospital stay in our institution.

Our study provides evidence that wound complications affect the time of initiating adjuvant treatments and hospitalization in patients with ovarian cancer, which may affect responses to chemotherapy and overall survival in these patients. ${ }^{5-7}$ However, there were no differences in progression free survival or overall survival between patients with wound complications and those with clear healing, which may be because of the small number of patients with wound complications or the short follow-up period. Therefore, additional data are needed to assess the effect of these delays on cancer survival. 


\section{Original research}

This study had inherent limitations, such as bias in patient selection, differences in surgical skills among surgeons, and its retrospective design. Despite these limitations, this study had several advantages: (1) all subcutaneous wound drains were placed by a single surgeon; thus, although it was a retrospective study, the wound drain placement was performed using a consistent and standardized method; (2) we included only patients who underwent primary ovarian cancer surgeries, with the exclusion of recurrent surgeries, because heterogeneity of surgery types could influence the results; and (3) we investigated the clinical benefits of clear wound healing, including the length of hospital stay and the time interval from surgery to initiation of adjuvant treatment.

In conclusion, despite multiple risk factors associated with an increased rate of wound complications, a lower rate of wound complications in group 1 than in group 2 suggested a benefit for subcutaneous negative pressure drains in reducing wound complications in patients undergoing open ovarian cancer surgeries. Therefore, we recommend these drains as a therapeutic intervention for the prevention of wound complications. While our results are encouraging, further investigations with large and prospective studies are needed.

Contributors Conception and design of the study was done by YSC, J-YL, EJN, SHK, SWK, and Y-TK. Data collection was done by S-HK and J-YL. Data analysis and interpretation was done by Y-TK and EJN. Statistical analysis was done by S-HK and Y-TK. Manuscript preparation was done by YSC and J-YL. Manuscript editing was done by YSC and S-HK. Patient recruitment was done by EJN and SWK. All authors read and approved the final manuscript.

Funding The authors have not declared a specific grant for this research from any funding agency in the public, commercial, or not-for-profit sectors.

Competing interests None declared.

Patient consent for publication Not required.

Ethics approval The study was conducted with the approval of the institutional review board of Severance Hospital, Yonsei University College of Medicine (No 4-2019-0900).

Provenance and peer review Not commissioned; externally peer reviewed.

Data availability statement All data relevant to the study are included in the article or uploaded as supplementary information.

\section{ORCID iD}

Jung-Yun Lee http://orcid.org/0000-0003-0297-3116

\section{REFERENCES}

1 Novetsky AP, Zighelboim I, Guntupalli SR, et al. A phase II trial of a surgical protocol to decrease the incidence of wound complications in obese gynecologic oncology patients. Gynecol Oncol 2014;134:233-7.

2 Nugent EK, Hoff JT, Gao F, et al. Wound complications after gynecologic cancer surgery. Gynecol Oncol 2011;121:347-52.

3 Kirkland KB, Briggs JP, Trivette SL, et al. The impact of surgicalsite infections in the 1990s: attributable mortality, excess length of hospitalization, and extra costs. Infect Control Hosp Epidemiol 1999;20:725-30.

4 Mahdi H, Gojayev A, Buechel M, et al. Surgical site infection in women undergoing surgery for gynecologic cancer. Int J Gynecol Cancer 2014;24:779-86.

5 Clark RM, Growdon WB, Wiechert A, et al. Patient, treatment and discharge factors associated with hospital readmission within 30 days after surgical cytoreduction for epithelial ovarian carcinoma. Gynecol Oncol 2013;130:407-10.

6 Tran CW, McGree ME, Weaver AL, et al. Surgical site infection after primary surgery for epithelial ovarian cancer: predictors and impact on survival. Gynecol Oncol 2015;136:278-84.

7 O'Donnell RL, Angelopoulos G, Beirne JP, et al. Impact of surgical site infection (SSI) following gynaecological cancer surgery in the UK: a trainee-led multicentre audit and service evaluation. BMJ Open 2019;9:e024853.

8 Gallup DC, Gallup DG, Nolan TE, et al. Use of a subcutaneous closed drainage system and antibiotics in obese gynecologic patients. Am J Obstet Gynecol 1996;175:358-62. discussion 362.

9 Hellums EK, Lin MG, Ramsey PS. Prophylactic subcutaneous drainage for prevention of wound complications after cesarean delivery--a metaanalysis. Am J Obstet Gynecol 2007;197:229-35.

10 Samraj K, Gurusamy KS. Wound drains following thyroid surgery. Cochrane Database Syst Rev 2007.

11 Kosins AM, Scholz T, Cetinkaya M, et al. Evidence-based value of subcutaneous surgical wound drainage: the largest systematic review and meta-analysis. Plast Reconstr Surg 2013;132:443-50.

12 Thomson DR, Sadideen H, Furniss D. Wound drainage after axillary dissection for carcinoma of the breast. Cochrane Database Syst Rev 2013.

13 Cardosi RJ, Drake J, Holmes S, et al. Subcutaneous management of vertical incisions with 3 or more centimeters of subcutaneous fat. Am J Obstet Gynecol 2006;195:607-14. discussion 614-606.

14 Magann EF, Chauhan SP, Rodts-Palenik S, et al. Subcutaneous stitch closure versus subcutaneous drain to prevent wound disruption after cesarean delivery: a randomized clinical trial. Am J Obstet Gynecol 2002;186:1119-23.

15 Ramsey PS, White AM, Guinn DA, et al. Subcutaneous tissue reapproximation, alone or in combination with drain, in obese women undergoing cesarean delivery. Obstet Gynecol 2005;105:967-73.

16 Panici PB, Zullo MA, Casalino B, et al. Subcutaneous drainage versus no drainage after minilaparotomy in gynecologic benign conditions: a randomized study. Am J Obstet Gynecol 2003;188:71-5.

$17 \mathrm{Kim}$ SI, Lim MC, Bae HS, et al. Benefit of negative pressure drain within surgical wound after cytoreductive surgery for ovarian cancer. Int J Gynecol Cancer 2015;25:145-51.

18 Coletta D, Del Basso C, Giuliani G, et al. Subcutaneous suction drains do not prevent surgical site infections in clean-contaminated abdominal surgery-results of a systematic review and meta-analysis. Langenbecks Arch Surg 2019;404:663-8.

19 Watanabe J, Ota M, Kawamoto $\mathrm{M}$, et al. A randomized controlled trial of subcutaneous closed-suction Blake drains for the prevention of incisional surgical site infection after colorectal surgery. Int $J$ Colorectal Dis 2017;32:391-8.

20 Aletti GD, Eisenhauer EL, Santillan A, et al. Identification of patient groups at highest risk from traditional approach to ovarian cancer treatment. Gynecol Oncol 2011;120:23-8.

21 Lee YJ, Lee JY, Cho MS, et al. Incorporation of paclitaxelbased hyperthermic intraperitoneal chemotherapy in patients with advanced-stage ovarian cancer treated with neoadjuvant chemotherapy followed by interval debulking surgery: a protocolbased pilot study. J Gynecol Oncol 2019;30:e3.

22 Webster J, Liu Z, Norman G, et al. Negative pressure wound therapy for surgical wounds healing by primary closure. Cochrane Database Syst Rev 2019;3.

23 Argenta LC, Morykwas MJ. Vacuum-assisted closure: a new method for wound control and treatment: clinical experience. Ann Plast Surg 1997;38:563-77. discussion 577.

24 Smits A, Lopes A, Das N, et al. Surgical morbidity and clinical outcomes in ovarian cancer - the role of obesity. BJOG 2016;123:300-8.

25 Wallace SK, Halverson JW, Jankowski CJ, et al. Optimizing blood transfusion practices through bundled intervention implementation in patients with gynecologic cancer undergoing laparotomy. Obstet Gynecol 2018;131:891-8.

26 Lundin ES, Johansson T, Zachrisson H, et al. Single-dose tranexamic acid in advanced ovarian cancer surgery reduces blood loss and transfusions: double-blind placebo-controlled randomized multicenter study. Acta Obstet Gynecol Scand 2014;93:335-44. 